\title{
Short-pulse UV laser ablation of solid and liquid metals: indium
}

\author{
T. Götz, M. Stuke \\ Max-Planck-Institut für biophysikalische Chemie, P.O.Box 2841, D-37018 Göttingen, Germany \\ (Fax: +49-551/2011330, E-mail: MSTUKE@gwdg.de)
}

Received: 2 December 1996/Accepted: 11 December 1996

\begin{abstract}
Laser-ablation experiments on solid $(T=300 \mathrm{~K})$ and liquid $(T=600 \mathrm{~K})$ indium are reported. The ablation was performed under high vacuum conditions with UV laser pulses at $248 \mathrm{~nm}$ and pulse durations of $15 \mathrm{~ns}$ and $0.5 \mathrm{ps}$. The ablated neutral indium atoms were resonantly ionized with a second laser pulse $28 \mathrm{~mm}$ above the sample surface and detected in a time-of-flight mass spectrometer. The ablation threshold fluence for solid indium decreases by a factor of 40 from $100 \mathrm{~mJ} / \mathrm{cm}^{2}$ to $2.5 \mathrm{~mJ} / \mathrm{cm}^{2}$ when a $0.5 \mathrm{ps}$ pulse is used instead of a $15 \mathrm{~ns}$ laser pulse. Measurements on liquid indium show a different behavior. With $15 \mathrm{~ns}$ laser pulses the threshold fluence is lowered by a factor of $\sim 3$ from $100 \mathrm{~mJ} / \mathrm{cm}^{2}$ for solid indium to $30 \mathrm{~mJ} / \mathrm{cm}^{2}$ for liquid indium. In contrast, measurements with $0.5 \mathrm{ps}$ laser pulses do not show any change in the ablation threshold and are independent of the phase of the metal at $2.5 \mathrm{~mJ} / \mathrm{cm}^{2}$. This behavior could be explained by thermal diffusion and heat conduction during the laser pulse and demonstrates in an independent way the energy lost into the material when long laser pulses are applied. Time-of-flight measurements to investigate the underlying ablation mechanism show thermal behavior of the ablated indium atoms for both ps and ns ablation and can be fitted to Maxwell-Boltzmann distributions.
\end{abstract}

PACS: 78.66.Bz; 42.62.-b; 81.15.Fg

The interaction of laser light with materials and the processing of surface modifications have been investigated for many years. Nevertheless the study of desorption and ablation processes is still a growing field in basic science, engineering, and micro-mechanics [1-6]. The study of the basic desorption and ablation mechanisms is a great challenge, since the underlying processes are still not completely understood. On the other hand, laser material treatment is applied in very different fields, especially in micro-machining and micro-fabrication. Obviously, laser ablation depends on a large number of parameters such as the material properties, laser parameters, and chemical environment. Therefore, the investigation of the suitable regimes for laser treatment of different materials is of high priority. Current laser ablation experiments concern semiconductors [7], materials such as crystals and glasses [8-10] that are transparent in the visible, polymers $[11,12]$, and metals $[13,14]$. The precise ablation of metals presents some difficulties, since the high thermal conductivity of metals results in a large energy loss by heat diffusion into the bulk during the laser pulse. This dissipating thermal energy leads to an increasing temperature of the material outside the laser spot, not resulting in ablation but modification of the crystal structure. For this reason, it is important to reduce the energy loss and heat transfer into the material, to prevent undesired modifications, and to search for new nonthermal, i.e. electronic, ablation mechanisms.

One possibility for reducing the energy loss into the material is to shorten the laser pulse duration to the femtosecond region. For fs laser pulses the heat diffusion into the material is negligible and, as a consequence, the energy loss during the laser pulse is minimized $[15,17]$. The thermal diffusion length $L_{\mathrm{th}}$ is given by $L_{\mathrm{th}} \propto\left(\kappa \tau_{\mathrm{p}}\right)^{1 / 2}$, were $\kappa$ is the thermal diffusivity and $\tau_{\mathrm{p}}$ is the pulse duration. The thermal diffusivity is related to the thermal conductivity $k$ by $\kappa=k / \varrho c$, were $\varrho$ is the density and $c$ is the heat capacity of the metal [16]. For 15-ns pulses the thermal diffusion length for metals is of the order of $1 \mu \mathrm{m}$ and decreases to about $20 \mathrm{~nm}$ for 0.5 -ps pulses [17]. Consequently, rapid energy accumulation occurs in the optical absorption volume for fs pulses and hence efficient ablation takes place if the accumulated energy reaches a critical value, of the order of the enthalpy of evaporation. In other words, compared to the case with ns laser pulses, the ablation threshold is drastically lowered for short laser pulses. To measure the influence of the thermal heat diffusion on the ablation process we have investigated the ablation threshold fluence directly by changing the laser pulse duration from $15 \mathrm{~ns}$ to $0.5 \mathrm{ps}$. A second and independent way to show the influence of energy loss by heat diffusion into the material is, according to the relation $L_{\mathrm{th}} \propto\left(\kappa \tau_{\mathrm{p}}\right)^{1 / 2}$, to vary the thermal properties, i.e. the thermal diffusivity $\kappa$ or the thermal conductivity $k$ at a constant laser pulse duration. Since the thermal conductivity for indium at the melting point changes in a step-like function by a factor of 2 [18-20], this must influence the thermal diffusion length and hence the energy loss into the material during the laser pulse. 
This dramatic change in the thermal conductivity to a lower value must result in a lowering of the threshold fluence.

To obtain further information about the basic mechanisms of metal ablation it is necessary to perform measurements at extremely low ablation rates, i.e. ablation rates much less than 1 monolayer. In this case no interaction between the ablated species through collisions, shock waves, and plasma formation can take place. This allows us to measure the velocity and angular distribution [21] of the ablated species resulting from the initial ablation process, and thus to distinguish between thermal and nonthermal processes [22-25]. In our experiment we have investigated the time-of-flight distribution of the ablated indium atoms from solid and liquid indium samples by using UV laser pulses in the ns and fs regimes.

\section{Experimental}

The experimental arrangement is schematically shown in Fig. 1. All the experiments reported in this paper were performed under high vacuum conditions at a pressure of $10^{-7}$ mbar. The sample consists of an indium disk, $35 \mathrm{~mm}$ in diameter and $5 \mathrm{~mm}$ thick. It was prepared by melting highpurity indium (99.99\%) in a glass crucible. The absolutely smooth and highly reflecting metallic surface is illuminated with a laser beam perpendicular to the indium surface. To guarantee that during the measurement every laser pulse illuminates a new surface area, which has not been modified by previous laser pulses, the indium sample can be rotated by a motor. Furthermore, the sample can be heated to $700 \mathrm{~K}$. This allows measurements on solid and liquid indium under identical conditions. The sample temperature is measured by a thermocouple. A commercial excimer laser (Lambda Physik), operating at a wavelength of $248 \mathrm{~nm}$, is used as the ablation laser for the measurement with 15 -ns pulse duration. The experiments with 0.5 -ps pulse duration are performed with a two-channel hybrid excimer laser at $248 \mathrm{~nm}$ [26]. The repetition rate of the system is $10 \mathrm{~Hz}$. The homogeneous part of the laser beam profile is used for the ablation experiments. The light focused onto the indium sample by means of a suprasil lens $(f=25 \mathrm{~cm})$ has a spot size on the indium surface of about $300 \mu \mathrm{m}$ in diameter. In front of the window of the vacuum chamber, a dielectric beam splitter is used to reflect $50 \%$ of the laser energy onto an energy meter (Polytec Rj 7200). This arrangement allows us to measure the energy of each pulse and to correlate the ablation signal with the pulse energy. This is important because small fluctuations in the laser intensity result in drastic changes in the ablation signal intensity. Furthermore, we can make direct fluence variations by attenuating the laser beam. We use a variable dielectric attenuator to change the fluence on the sample between $4 \%$ and $80 \%$ of the available energy of the laser. The beam line and hence the position of the spot are not affected by variations of the laser intensity because of the presence of a second compensating quartz plate.

A pulsed narrow-band doubled dye laser operating at $214 \mathrm{~nm}$ is used to ionize the ablated neutral indium atoms. Resonant ionization occurs after a flight distance of $28 \mathrm{~mm}$ above the sample, between the grids of a time-of-flight mass spectrometer. In the drift tube the indium ions are mass selected and detected by a channel plate. The detected signal is proportional to the number of indium atoms in the ionization volume. The signal is recorded by a digital signal analyzer and stored in a computer.

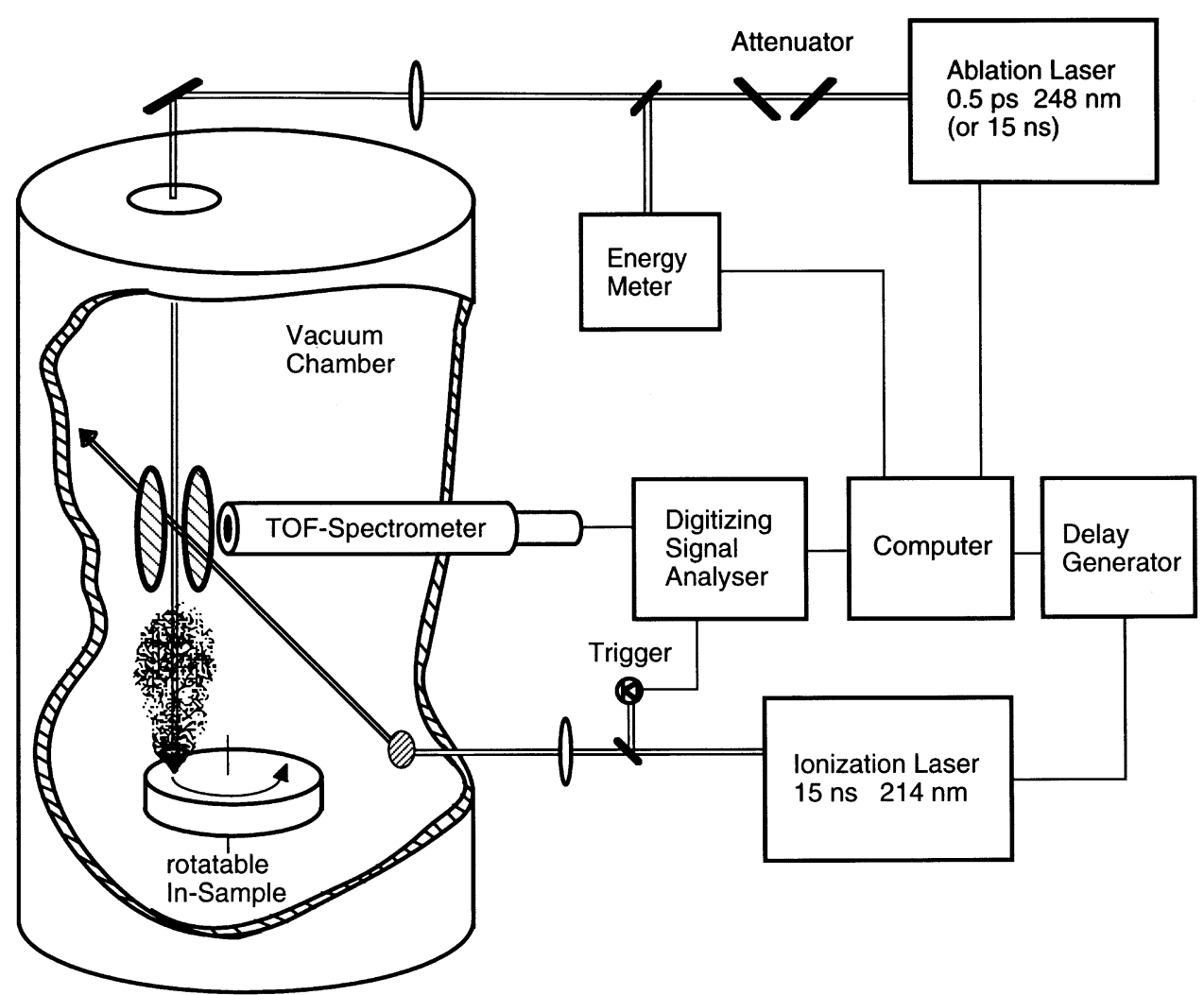

Fig. 1. Schematical sketch of the experimental setup 
Two different kinds of measurements were taken. First, the ablation threshold and the dependence of the ablation signal on the laser fluence were analyzed. To do this, a constant delay time between the ablation and ionization lasers is chosen and the fluence of the ablation laser is varied by the attenuator. Second, the ablation signal for a specific constant laser fluence for different delay times between the ablation and ionization lasers was recorded. This measurement allows us to determine the time-of-flight distribution and hence the kinetic energy of the ablated indium atoms. From these measurements it is possible to extract details of the underlying ablation mechanism.

\section{Experimental results}

The ablation threshold and ablation signal as a function of the laser fluence were measured for solid and liquid indium for 15-ns and 0.5-ps pulse durations at a laser wavelength of $248 \mathrm{~nm}$. The delay time between the ablation and ionization lasers was set to $24 \mu \mathrm{s}$. The largest signal was detected at this delay time and thus the highest sensitivity for the ablation threshold measurement was obtained.

Figure 2 shows the ablation signal of indium atoms, emitted as neutral atoms in perpendicular direction from the solid indium surface, as a function of the laser fluence for 15-ns and 0.5-ps pulses. For 15-ns pulses the ablation threshold is about $100 \mathrm{~mJ} / \mathrm{cm}^{2}$. The signal increases slightly with the fluence. Above $120 \mathrm{~mJ} / \mathrm{cm}^{2}$ the signal increases drastically and shows a linear behavior in the investigated fluence regime. The ablation with 0.5 -ps pulses also shows, under exactly equal conditions, a linear dependence, but in contrast to the case for ablation with 15-ns laser pulses, the threshold fluence is now lowered by a factor of 40 down to $2.5 \mathrm{~mJ} / \mathrm{cm}^{2}$. Furthermore, the slope of the linear increase for fs ablation is 3.5 times higher than for ns ablation. For liquid indium the ablation threshold for ns ablation is lowered by a factor of 3 compared to the threshold in the solid phase (Fig. 3), indicating that the physical properties responsible for ablation do

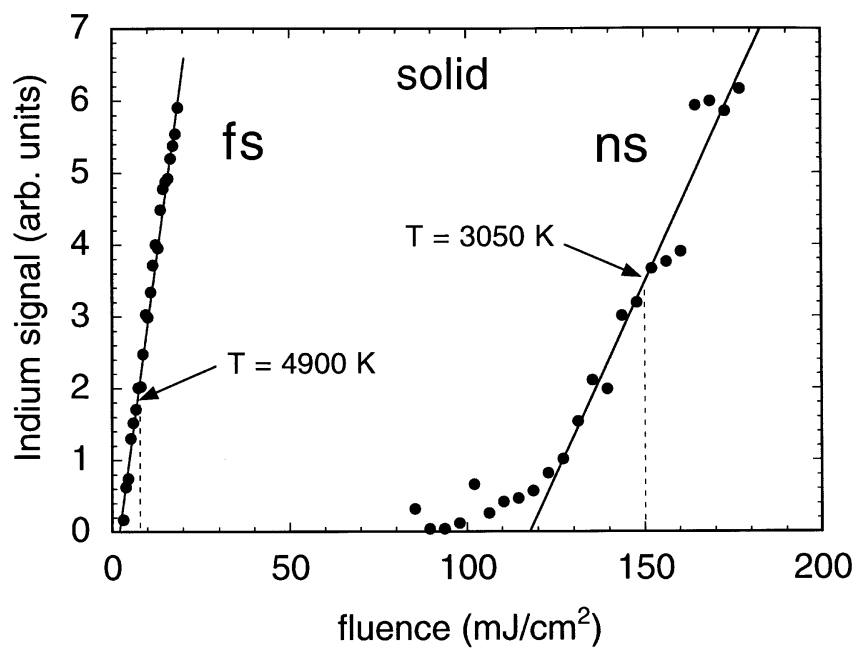

Fig. 2. Ablation signal of the detached neutral indium atoms from solid indium as a function of laser fluence for short (500-fs) and long (15-ns) laser pulses at $248 \mathrm{~nm}$. The ablation threshold for 500 -fs laser pulses is lowered by a factor of 40 compared to the ablation threshold for 15-ns laser pulses. The translation temperature of the atoms at the indicated laser fluence is deduced from the time-of-flight distribution (see also Fig. 5) change at the phase transition. The slope of the linear function of the ablation rate is nearly the same for both phases. For fs ablation, however, the ablation threshold fluence for liquid indium is identical to the threshold fluence for solid indium at $2.5 \mathrm{~mJ} / \mathrm{cm}^{2}$ (Fig. 4) except that the slope of the ablation signal for the liquid phase is increased by a factor of 3 compared to the solid ablation case.

Two examples of time-of-flight measurements for 15-ns ablation at $150 \mathrm{~mJ} / \mathrm{cm}^{2}$ and $0.5-\mathrm{ps}$ ablation at $7.35 \mathrm{~mJ} / \mathrm{cm}^{2}$ for solid indium are shown in Fig. 5. The measurements can be approximated by Maxwell-Boltzmann velocity distributions. The translational temperatures are $4900 \mathrm{~K}$ for fs ablation at a fluence 20 times less than that for ns ablation, for which a temperature of $3050 \mathrm{~K}$ is deduced. Both the fluences and the temperatures, are indicated in Fig. 2. Additional time-offlight measurements show for ns and fs ablation an increase

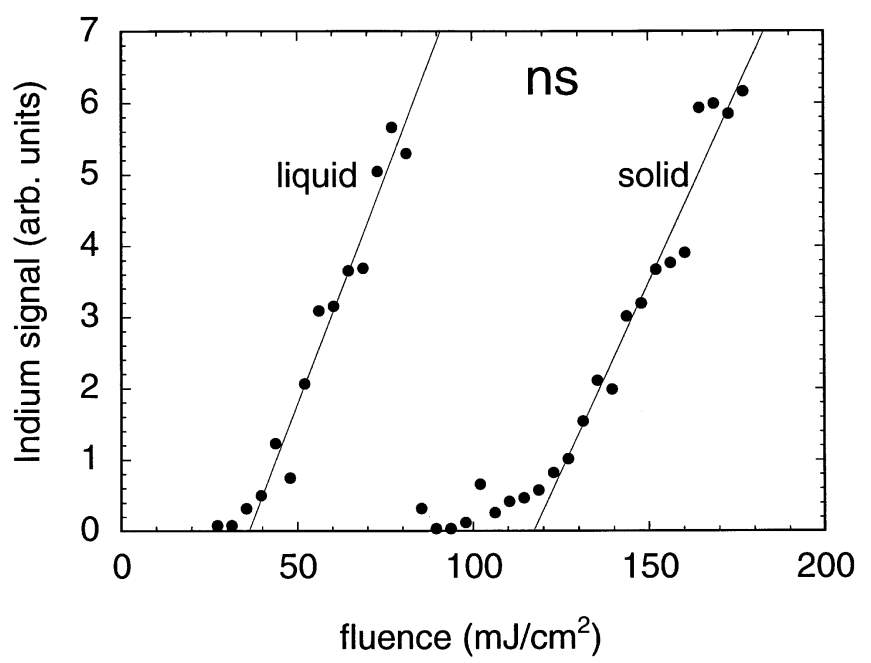

Fig. 3. Ablation signal of the detached neutral indium atoms from solid and liquid indium after illumination with 15 -ns laser pulses at a 248 -nm wavelength. The ablation threshold is dependent on the liquid or solid state of the indium metal. In case of liquid indium the threshold is lowered by a factor of 3 compared to the solid state

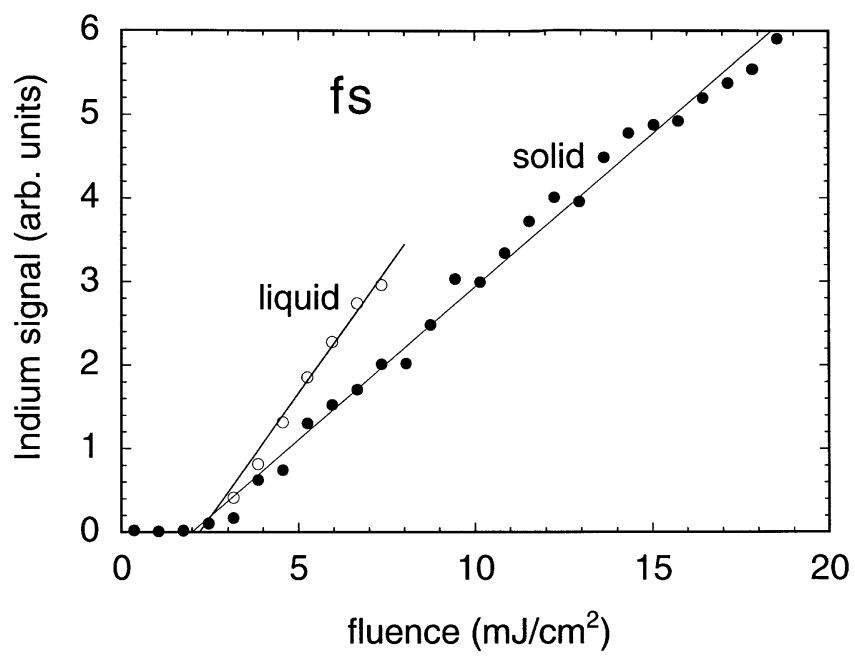

Fig. 4. Ablation signal of the detached neutral indium atoms from liquid and solid indium after illumination with 500-fs laser pulses at a $248-\mathrm{nm}$. The ablation threshold is independent of the liquid or solid state of the indium metal. Above the threshold, the ablation is more efficient for liquid indium 


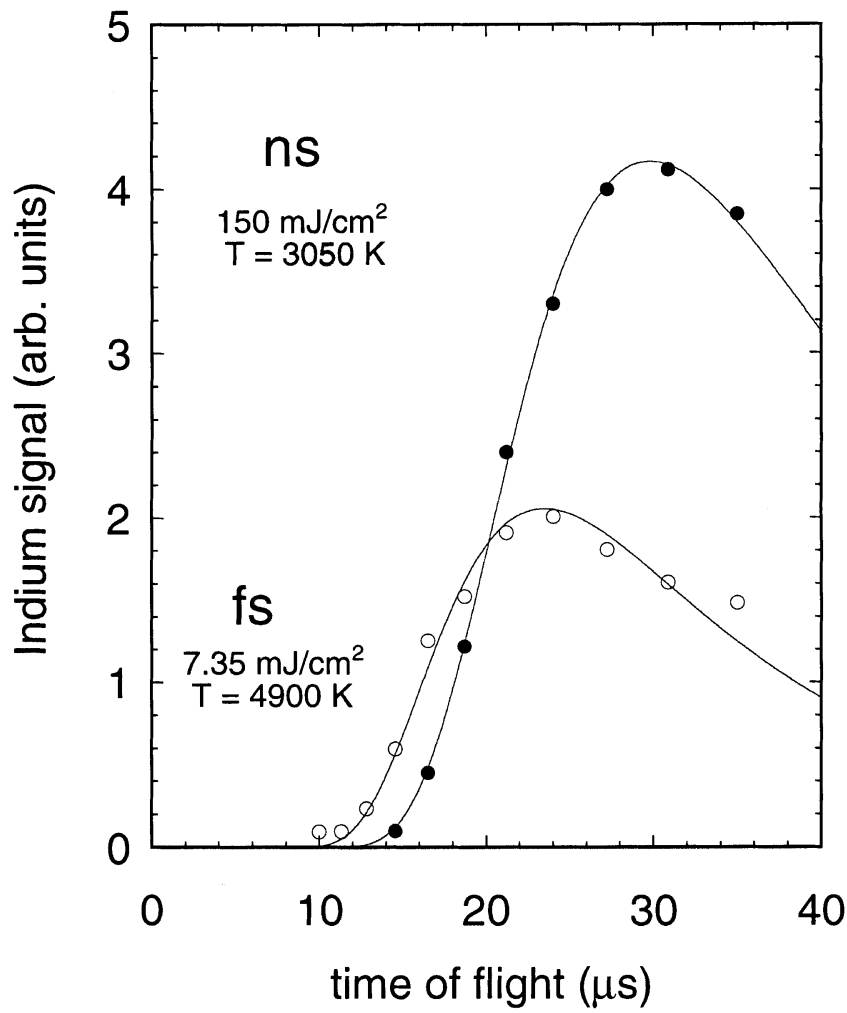

Fig. 5. Time-of-flight distribution of the indium atoms detached from solid indium for 500 -fs and 15 -ns laser pulses. The measured values are fitted with a Maxwell-Boltzmann distribution resulting in translation temperatures of $3050 \mathrm{~K}$ and $4900 \mathrm{~K}$, respectively (see also Fig. 2)

in both the ablation rate and the translational temperature of the ablated atoms with an increasing fluence. Obviously, the accumulated energy for removing the same amount of material from the surface, i.e. the same signal intensity in the time-offlight spectrometer, by using fs pulses results in ablated atoms with higher energy compared to atoms emitted by ns pulses.

\section{Discussion}

We define here the ablation efficiency $\eta$ as the ratio of the number of ablated atoms $\left(N_{\text {atom }}\right)$ to the number of photons $\left(N_{\text {photon}}\right)$ illuminating (not absorbed by) the surface $\left(\eta=N_{\text {atom }} / N_{\text {photon }}\right)$. Since the fluence is proportional to the number of photons and the channeltron signal is proportional to the number of the ablated atoms, it is clear from Fig. 2 that fs laser pulses are much more efficient for the ablation process than ns pulses. With the increasing efficiency for short laser pulses, the thermal load on the surrounding material is less than for long laser pulses since the absolute energy deposited in the material is also lower. In other words, less energy is needed to remove the same amount of material with short laser pulses.

The higher ablation threshold for ns laser pulses is caused by the energy dissipation into the material during the long laser pulse, whereas during the short laser pulse a negligible energy dissipation into the material occurs, resulting in a drastically lower ablation threshold.

The most striking difference between fs and ns ablation of solid and liquid indium is the fact that for fs irradiation the ablation threshold is independent of the metal phase, whereas for ns ablation the ablation thresholds for solid and liquid indium are different. This behavior occurs not only for the measurement at the delay time of $24 \mu \mathrm{s}$ between the ablation and ionization laser presented in Fig. 2, 3 and 4 but also for all other delay times. For this reason it must correlate with a specific change in the physical properties of the indium sample during the transition from solid to liquid. For example, the optical penetration depth, relaxation time, or other optical properties, such as reflection, change slightly at the transition from the solid to the liquid phase. But since these changes are observed for both time regimes, ns and fs, in the same way - because the applied fluence is too low for two-photon processes - the ablation behavior should also change for ns and fs pulse in the same way.

We suggest the following mechanism to explain the different behavior. In the solid indium the light is absorbed within the optical penetration depth of about $20 \mathrm{~nm}$ by electron excitation. The excited electrons thermalize in a very short time, typically of the order of $10^{-14} \mathrm{~s}$, to a thermal electron distribution. This energy relaxes by electron-phonon coupling on a time scale of $10^{-12} \mathrm{~s}$ to the lattice, resulting in an energy transfer to the atoms and heating of the material [27]. If the energy necessary to release atoms from the surface is reached, desorption occurs. This energy is accumulated much faster for fs pulses than for ns pulses since the energy loss by dissipation through thermal conductivity, according to $L_{\mathrm{th}} \propto\left(\kappa \tau_{\mathrm{p}}\right)^{1 / 2}$, is minimized for fs pulses. This is well known and has been shown for metals and other materials.

In the case of liquid indium the excitation mechanism is unchanged, but since the lattice structure is no longer present, the thermal conductivity changes drastically at the phase transition from the solid to the liquid phase. The thermal conductivity of liquid indium changes in an extremely small temperature region around the melting point at $429 \mathrm{~K}$ from $78 \mathrm{~W} / \mathrm{mK}$ for solid indium to $40 \mathrm{~W} / \mathrm{mK}$ for liquid indium [18-20]. As a result, the energy dissipation by thermal conductivity is much lower than in the solid phase. This, for the case of ns laser pulses, leads to a larger energy accumulation close to the surface at lower fluences, and hence to a reduction of the ablation threshold. For fs irradiation the thermal conductivity changes in the same way, but since during the 0.5 ps laser pulse almost no energy dissipation occurs - for both phases the thermal diffusion length is on the order of a few nm, which is within the optical penetration depth - thermal conductivity can be neglected and hence the ablation occurs at the same fluence threshold as for the solid phase (see Fig. 4). These measurements for solid and liquid indium for ns and fs laser pulses show in an independent way - by varying the thermal conductivity of the indium metal - the influence of the energy loss through diffusion during the laser pulse duration.

The different slopes of the ablation functions can be explained by the following considerations. The ablation rate detected in this experiment is kept to less than one monolayer per laser pulse and the ablated atoms originate from locations on the surface with special binding sites or geometric orientations. This can be concluded from the fact that the ablation rate decreases as a function of the number of applied laser pulses if, in consecutive pulses, the same indium surface is illuminated. In other words, the laser modifies the surface in a way that, at a constant fluence, all atoms with a specific, i.e. low, binding energy are removed, or the surface is modified by rear- 
rangement of the surface atoms. Solid and liquid indium have definitely different surface nano-structures, because the lattice structure is lost, i.e. the number of atoms that can be ablated at a constant fluence is different. Since the ablation for fs laser pulses occurs in a very short time (the energy is accumulated very rapidly), the ablation rate gives information about the number of these sites, because these sites are 'frozen' during this time. For the liquid state the number of atoms at specific sites is higher than that for the solid state and hence the slope of the ablation signal is higher. For ns ablation the energy in the sample accumulates in a much longer time; the pulse duration is $3 \times 10^{4}$ times longer than for $\mathrm{fs}$ ablation and hence the surface reaches the ablation temperature much later. During this time the surface can be rearranged for the solid as well as for the liquid phase by surface diffusion of the atoms, so that in both cases an almost identical surface structure can be reached and hence the same number of atoms can be ablated. This is the reason for the nearly equal slopes in the case of ns ablation.

The time-of-flight distribution for both time regimes and material phases can be fitted to Maxwell-Boltzmann distributions (Fig. 5). This is not contradictory to a thermal ablation mechanism.

\section{Conclusion}

A detailed study of the ablation process of solid and liquid indium with UV laser light is reported for the first time. For the solid sample a reduction in the ablation threshold is observed if the pulse duration is reduced from ns to fs. This reduction is explained by a reduced heat diffusion during the short pulse. For liquid indium no threshold reduction compared to solid indium is observed for fs pulses, because the change in the thermal conductivity does not affect the thermal diffusion during the short-pulse duration. In contrast, we observe for ns irradiation a reduction in the threshold fluence compared to the solid phase, since the lower thermal diffusion allows a better energy accumulation close to the surface. The measurements on solid and liquid indium show, the influence of the thermal diffusion on the ablation process. Further experiments with other metals will be performed to demonstrate the general behavior of this effect. Furthermore we have shown by time-of-flight measurements that the ablation mechanism is thermal.

Acknowledgements. We thank K. Müller for expert technical assistance and BMBF 13N 61597 for financial support.

\section{References}

1. AIP Conference Proceedings 288 Laser Ablation: Mechanisms and Applications-II (1994)

2. E. Fogarassy, D. Geohegan, M. Stuke (Eds.): Proceedings of Symposium F: Third International Conference on Laser Ablation (COLA'95) of the 1995 E-MRS Spring Conference (1995) Appl. Surf. Sci. 96-98, (1996)

3. J.C. Miller: Laser Ablation Springer Series in Material Science 28 (Springer, Berlin, Heidelberg 1994)

4. D.Bäuerle Laser Processing and Chemistry, 2nd ed. (Springer, Berlin, Heidelberg 1996)

5. M.v. Allmen, A. Blatter: Laser-Beam Interactions with Materials Springer Series in Material Science (Springer, Berlin, Heidelberg 1995)

6. I.W. Boyd: Laser Processing of Thin Films and Microstructures Springer Series in Material Science 3 (Springer, Berlin, Heidelberg 1987)

7. R.F. Haglund, N. Itoh: in [3]

8. E. Matthias, H.B. Nielsen, J. Reif, A. Rosen, E. Westin: J. Vac. Sci. Technol. B5, 1415 (1987)

9. Th. Beuermann, H.J. Brinkmann, T. Damm, M. Stuke: Mat. Res. Soc. Symp. Proc. 191, (1990)

10. J.T. Dickinson, L.C. Jensen, R.L. Webb, M.L. Dawes, S.C. Langford: J. Appl. Phys. 74, 3758 (1993)

11. R. Srinivasan, V. Mayne-Banton: Appl. Phys. Lett. 41, 576 (1982)

12. S. Küper, S. Modaressi, M. Stuke: J. Phys. Chem. 94, 7514 (1990)

13. T. Götz, M. Bergt, W. Hoheisel, F. Träger, M. Stuke: Appl. Phys. A 63, 315 (1996)

14. S. Preuss, A. Demchuk, M. Stuke: Appl. Phys. A 61, 33 (1995)

15. E. Matthias, M. Reichling, J. Siegel, O.W. Kädig, S. Petzoldt, H. Skurk, P. Bitzenberger, E. Neske: Appl. Phys. A 58, 129 (1994)

16. A. Rosencwaig: Photoacoustics and Photoacoustic Spectroscopy Chemical Analysis Vol. 57 (Wiley, New York 1980)

17. S. Preuss, E. Matthias, M. Stuke: Appl. Phys. A 59, 83 (1994)

18. E. Goldratt, A.J. Greenfield: J. Phys. F 10, L95 (1980)

19. M.J. Duggin: J. Phys. F 2, 433 (1972)

20. B.P. Pashayev, A.M-A. Magomedov: Heat Transfer-Sov. Res. 5, 1 (1973)

21. P.D. Brewer, M. Späth, M. Stuke: Mat. Res. Soc. Proc. Vol. 334, 245 (1994)

22. T. Götz, M. Bergt, W. Hoheisel, M. Stuke, F. Träger: Appl. Surf. Sci. 96-98, 280 (1996)

23. I. Lee, T.A. Callcott, E.T. Arakawa: Phys. Rev. B 47, 6661 (1993)

24. H.T. Liu, Z. Wu: Phys. Rev. Lett. 72, 3891 (1994)

25. A.M. Bonch-Bruevich, T.A. Vartanyan, A.V. Gorlanov, Yu.N. Maksimov, S.G. Przhibelskii, V.V. Khromov: Sov. Phys. JETP 70, 604 (1990)

26. G. Almasi, S. Szatmari, P. Simon: Opt. Comm. 88, 231 (1992)

27. S.I. Anisimov, V.A. Khokholov: Instabilities in Laser-Matter Interaction (CRC Press 1995) 